\title{
Review
}

\section{Multiple system atrophy: clinicopathological characteristics in Japanese patients}

\author{
By Tetsutaro OzawA* ${ }^{* 1, \dagger}$ and Osamu OnODERA ${ }^{* 2}$ \\ (Communicated by Kunihiko SuzukI, M.J.A.)
}

\begin{abstract}
Multiple system atrophy (MSA) is an adult-onset neurodegenerative disorder that has both clinical and pathological variants. Clinical examples include MSA with predominant cerebellar ataxia (MSA-C) and MSA with predominant parkinsonism (MSA-P), whereas olivopontocerebellar atrophy and striatonigral degeneration represent pathological variants. We performed systematic reviews of studies that addressed the relative frequencies of clinical or pathological variants of MSA in various populations to determine the clinicopathological characteristics in Japanese MSA. The results revealed that the majority of Japanese patients have MSA-C, while the majority of patients in Europe and North America have MSA-P. A comparative study of MSA pathology showed that the olivopontocerebellar-predominant pathology was more frequent in Japanese MSA than in British MSA. Demonstrated differences in pathological subtype thus appear consistent with differences in the clinical subtype of MSA demonstrated between Japan and European populations. We concluded that olivopontocerebellar-predominant pathology and MSA-C may represent clinicopathological characteristics in Japanese MSA. Factors determining predominant involvement of olivopontocerebellar regions in MSA should therefore be explored.
\end{abstract}

Keywords: multiple system atrophy, Parkinson's disease, spinocerebellar ataxia, genetic background, environmental factor

\section{Introduction}

Multiple system atrophy (MSA) is a progressive neurodegenerative disease clinically characterized by autonomic failure in addition to various combinations of parkinsonism, cerebellar ataxia, and pyramidal dysfunction. ${ }^{1)}$ In 1969, Graham and Oppenheimer first introduced the term MSA to encompass the pathological entities of olivopontocerebellar ataxia, striatonigral degeneration, and Shy-Drager

*1 Department of Neurology, Uonuma Institute of Community Medicine, Niigata University Medical and Dental Hospital, Niigata, Japan.

*2 Department of Neurology, Brain Research Institute, Niigata University, Niigata, Japan.

$\dagger$ Correspondence should be addressed: T. Ozawa, Department of Neurology, Uonuma Institute of Community Medicine, Niigata University Medical and Dental Hospital, 4132 Urasa, Minami-Uonuma, Niigata 949-7302, Japan (e-mail: ozawa@bri. niigata-u.ac.jp).

Abbreviations: MSA: Multiple system atrophy; OPC: olivopontocerebellar; $\mathrm{SN}$ : striatonigral; GWAS: genome-wide association study. syndrome on the basis of the neuropathological findings in these disorders. ${ }^{2)}$ Papp and Lantos subsequently discovered oligodendroglial cytoplasmic inclusions in the vulnerable regions of MSA, ${ }^{3)}$ which have been recognized as the cardinal neuropathological hallmark of the disease irrespective of clinical presentation. Aggregations of phosphorylated $\alpha$ synuclein were then found to represent a major constituent of oligodendroglial cytoplasmic inclusions, ${ }^{4)}$ leading to the notion that MSA involves a pathogenic mechanism similar to that of Parkinson's disease or dementia with Lewy bodies.

Consensus criteria for the clinical diagnosis of MSA currently classify the disorder into two subtypes: MSA-C, characterized by a predominance of cerebellar ataxia; and MSA-P, characterized by a predominance of parkinsonism. ${ }^{1}$ Neuropathological analyses of brain tissue from cases of MSA have identified MSA cases with predominant olivopontocerebellar (OPC) pathology and others with predominant striatonigral (SN) pathology, showing reasonable correlations with clinical findings.5),6) 
Furthermore, accumulating evidence from structuralfunctional imaging in patients with MSA strongly suggests that the OPC region is predominantly involved in MSA-C patients, whereas the $\mathrm{SN}$ region of the brain is predominantly involved in MSA-P patients during life. However, reasons for these differing emphases on $\mathrm{OPC}$ or $\mathrm{SN}$ involvement in MSA remain to be determined. To facilitate identification of susceptibility factors for MSA, differences in clinical and pathological characteristics of MSA between ethnic groups and populations should be elucidated.

This review provides a systematic overview of clinical and pathological studies of MSA that included large patient cohorts in various populations, to compare the relative frequencies of clinical and pathological subtypes of MSA between different ethnic groups. Furthermore, we summarized current hypotheses regarding the factors responsible for differences in MSA subtype distributions across populations.

\section{Methods}

A systematic review of clinical studies. To obtain information regarding the relative frequencies of MSA-C and MSA-P in different populations, we searched PubMed for studies that targeted large patient cohorts and were published after the first consensus criteria were released in 1999. ${ }^{7)}$ The following search formula was used for searching PubMed: ("multiple system atrophy"[MeSH Terms] AND "humans"[MeSH Terms] AND "1999"[Date Publication]: "3000”[Date - Publication] AND "English"[Language]) AND ("clinical study"[Publication Type] OR "observational study"[Publication Type] OR "multicenter study"[Publication Type] OR "comparative study"[Publication Type] OR "casecontrol") NOT ("case reports"[Publication Type] OR "letter"[Publication Type]). Abstracts of hit articles were reviewed to identify studies of natural history or epidemiological issues of MSA that included more than 100 consecutive patients. Studies that had settled purposes of recruiting patients with only a certain phenotype, such as MSA-C or MSA-P, were excluded. Studies including patients with other parkinsonian disorders or cerebellar ataxia were also excluded, along with studies focusing on specific symptoms, therapies, or laboratory data. Where more than two studies were conducted using a similar patient cohort or data set, only the most recent publication was included.
A systematic review of pathological studies. To obtain information regarding the relative prevalences of OPC- and SN-predominant cases in different populations, we searched PubMed for studies that targeted large cohorts of autopsied cases of MSA that had been published after the first consensus criteria were published in 1999. ${ }^{7}$ The following search formula was used for searching PubMed: ("multiple system atrophy"[MeSH Terms] AND "humans"[MeSH Terms] AND "1999"[Date Publication]: "3000”[Date - Publication] AND "english"[Language] AND "pathology") NOT ("case reports"'[Publication Type] OR "letter"[Publication Type]). Abstracts were reviewed to identify studies of quantitative or semi-quantitative analyses of pathology performed on more than 50 cases carried out using a well-defined grading system for both OPC and SN pathologies. To compare the relative frequencies of OPC- and SN-predominant cases between populations under fixed conditions, we further narrowed the search to those studies carried out under identical methods for quantitative or semiquantitative analyses of MSA pathology.

\section{Results}

Relative frequency of MSA-C and MSA-P in different populations. Using the search formula for systematic review of clinical studies, a total of 408 items were found in PubMed on December 6, 2016. According to the inclusion and exclusion criteria for reviewing abstracts mentioned above, we identified 8 studies involving more than 100 patients with possible or probable MSA, which addressed the relative frequencies of MSA-C and MSA-P. The breakdowns of MSA-P and MSA-C in each study are summarized in Table 1. In Japan, two large studies focused on the relative frequencies of MSA-C and MSA-P. From the northern island of Japan, a study involving 142 Japanese patients with MSA reported that 119 cases $(83.8 \%)$ were MSA-C and $23(16.2 \%)$ were MSA-P. ${ }^{8)}$ From the main island of Japan, 230 Japanese patients with MSA were examined, and the result was that 155 patients $(67.4 \%)$ had MSA-C and $75(32.6 \%)$ had MSA-P. ${ }^{9)}$ A study from Korea involving 100 Korean patients with MSA reported that MSA-C patients accounted for $73 \%$ of the cohort. ${ }^{10)}$ In Europe, the European MSA Study Group, involving research groups in 10 countries (Austria, Denmark, U.K., France, Germany, Israel, Italy, Portugal, Spain, and Sweden), recruited 437 European patients with MSA from 2001 to 2005. They reported that $68.2 \%$ of these patients were 
Table 1. Relative frequencies of clinical subtypes of MSA in Asia, Europe, and America

\begin{tabular}{|c|c|c|c|c|c|c|c|}
\hline Region & Author & Year & $\begin{array}{l}\text { Population } \\
\text { or regional } \\
\text { study group }\end{array}$ & $\begin{array}{l}\text { Total number of } \\
\text { patients }\end{array}$ & $\begin{array}{l}\text { Number of } \\
\text { MSA-C (\%) }\end{array}$ & $\begin{array}{l}\text { Number of } \\
\text { MSA-P (\%) }\end{array}$ & $\begin{array}{l}\text { Ref. } \\
\text { no. }\end{array}$ \\
\hline \multicolumn{8}{|l|}{ Asia } \\
\hline & Watanabe et al. & 2002 & Japan & 230 & $155(67.4)$ & $75(32.6)$ & 9 \\
\hline & Yabe et al. & 2006 & Japan & 142 & $119(83.8)$ & $23(16.2)$ & 8 \\
\hline & Seo et al. & 2010 & South Korea & 100 & $73(73)$ & $27(27)$ & 10 \\
\hline \multicolumn{8}{|l|}{ Europe } \\
\hline & Kollensperger et al. & 2010 & EMSASG & 437 & $139(31.8)$ & $298(68.2)$ & 11 \\
\hline & Wenning et al. & 2013 & EMSASG & 141 & $54(38.3)$ & $87(61.7)$ & 12 \\
\hline \multicolumn{8}{|l|}{ America } \\
\hline & Gatto et al. & 2014 & PANMSA & $159^{*}$ & $50(31.4)$ & $107(67.3)$ & 14 \\
\hline & & & & Caucasian: 107 & $30(20.8)$ & $\mathrm{NM}$ & \\
\hline & & & & Non-Caucasian: 52 & $27(51.9)$ & NM & \\
\hline & Low et al. & 2015 & U.S. & 175 & $49(28)$ & $126(72)$ & 13 \\
\hline
\end{tabular}

EMSASG, European MSA Study Group; PANMSA, Pan-American Consortium of MSA; U.S., United States; NM, not mentioned;

*Information of subtype was missing in 2 patients.

diagnosed with MSA-P and $31.8 \%$ were diagnosed with MSA-C. ${ }^{11)}$ The European MSA study group also undertook a prospective cohort study, which reduced the influence of selection biases determined by the clinical interest of the investigators in either parkinsonism or cerebellar dysfunction, and confirmed that the frequency of MSA-P (61.7\%) was higher than that of MSA-C $(38.3 \%)$ in Europe. ${ }^{12)}$ Looking at North America and Latin-American countries, two large studies were found in this systematic review. A study group in the United States performed a prospective cohort study involving 175 patients with MSA in which $93 \%$ of patients were non-Hispanic Caucasian. They reported that $72 \%$ were diagnosed with MSA-P and 28\% were diagnosed with MSAC. ${ }^{13)}$ The Pan-American Consortium of MSA published a large study of MSA in Argentina, Chile, Mexico, Peru, and the United States. ${ }^{14)}$ This is the first report of the relative frequency of MSA-C and MSA-P in patients in Latin American countries. In this study, 107 patients were Caucasian and 52 patients reported non-Caucasian ancestry. The MSA-C phenotype was identified in $20.8 \%$ of Caucasian individuals, and in $51.9 \%$ of non-Caucasian individuals. In that study, the relative frequencies of MSA-C and MSA-P in Caucasian individuals were similar to those reported in Western countries, with a predominance of MSA-P. However, interestingly, the MSA-C phenotype predominated in nonCaucasians, more specifically the Mestizo population, which is of non-white, mixed European, and Amerindian descent. ${ }^{14)}$ In non-Caucasian patients from
Latin American countries, MSA-C should be noted to predominate. In summary, in studies from Japan, Korea, and non-Caucasian populations in Latin America, the majority of patients exhibited features of MSA-C, whereas studies from Europe and North America showed a predominance of MSA-P patients.

Relative frequencies of OPC- and SNpredominant cases in different populations. Regarding search results from pathological studies, 603 items were found in PubMed on December 6, 2016. We reviewed abstracts of hit articles to narrow the search to those studies carried out under identical methods for quantitative or semi-quantitative analyses of MSA pathology in different patient cohorts. This review yielded only 2 studies that met the requirements, as follows. Ozawa et al. performed semi-quantitative assessments of neuronal cell loss at 24 anatomical sites in the $\mathrm{OPC}$ and $\mathrm{SN}$ regions (Fig. 1) in patients from the U.K. ${ }^{5)}$ and Japan ${ }^{6}$ using autopsied brain material from 100 British (Caucasian) cases of MSA referred to the Queen Square Brain Bank, London, U.K., and 50 cases of MSA referred to the Brain Research Institute, Niigata University, Japan. Results from these two isolated studies were summarized in a review article published in 2012. ${ }^{15)}$ They assessed severities of neuronal cell loss using sections stained by hematoxylin and eosin (HE) and Luxol fast blue/cresyl violet, and assigned one of four scores for the degree of neuronal loss $(0,1+, 2+$, or $3+)$ at each anatomical site (Fig. 1). ${ }^{15}$ ) The result showed a trend towards Japanese cases featuring greater involvement of the 


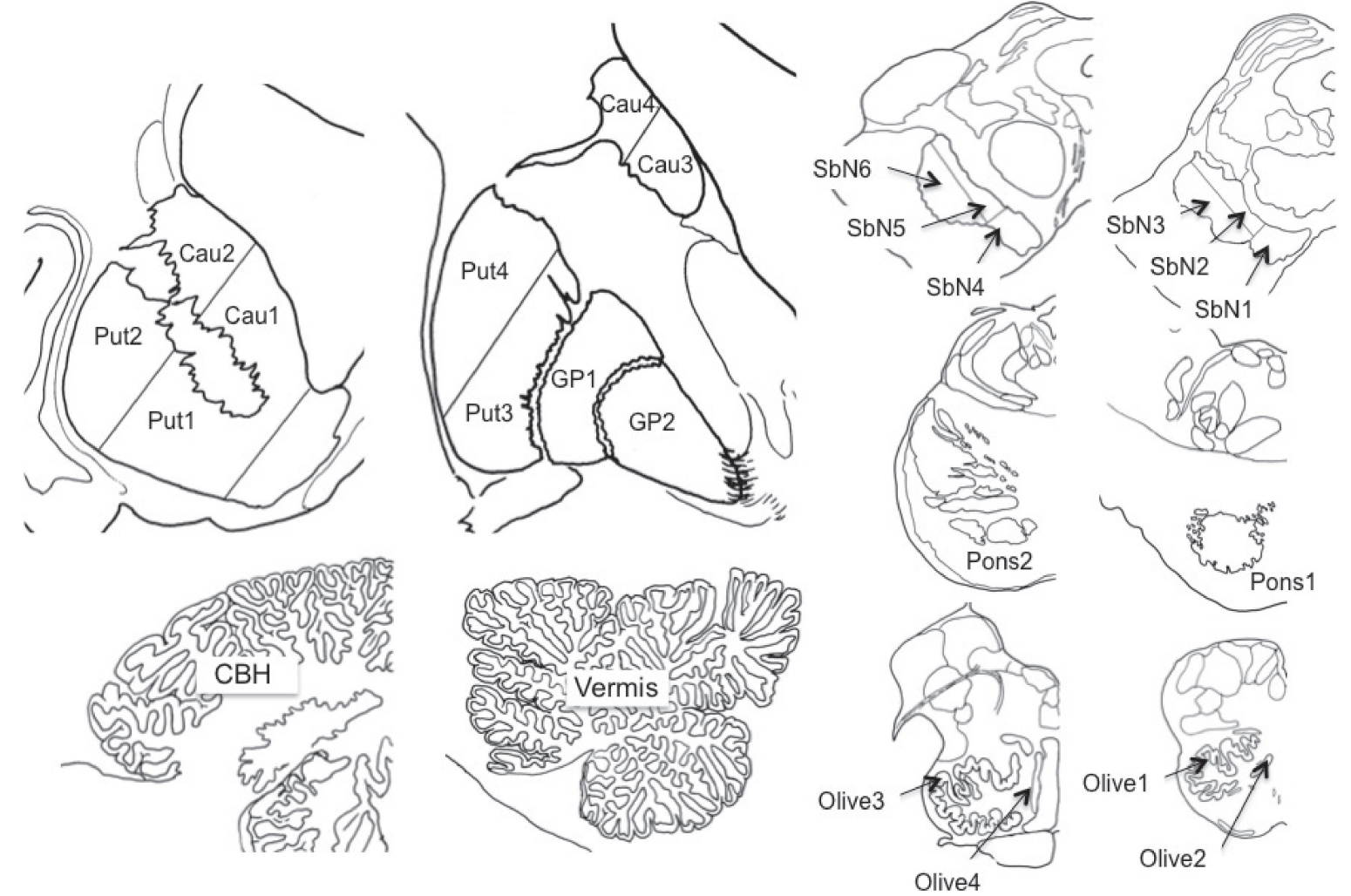

Fig. 1. The 24 anatomical sites in the striatonigral and olivopontocerebellar regions examined in a comparison of MSA cohorts in Japan and the U.K. Put $=$ putamen, Cau = caudate nucleus, $\mathrm{GP}=$ globus pallidus, $\mathrm{SbN}=$ substantia nigra, $\mathrm{CBH}=$ cerebellar hemisphere This figure was reprinted from the reference no. 15, with permission from IOS Press.

Table 2. Relative frequencies of pathological subtypes of MSA in Japan and U.K.

\begin{tabular}{|c|c|c|c|c|c|c|c|}
\hline Country & Author & Year & $\begin{array}{c}\text { Total } \\
\text { number } \\
\text { of cases }\end{array}$ & $\begin{array}{c}\text { Number of } \\
\text { OPC-predominant } \\
\text { pathology }(\%)\end{array}$ & $\begin{array}{c}\text { Number of } \\
\text { SN-predominant } \\
\text { pathology }(\%)\end{array}$ & $\begin{array}{l}\text { Number of OPC and } \\
\text { SN with equally } \\
\text { sever pathology }(\%)\end{array}$ & $\begin{array}{c}\text { Ref. } \\
\text { no. }\end{array}$ \\
\hline \multicolumn{8}{|l|}{ Japan } \\
\hline & Ozawa et al. & 2011 & 50 & $20(40)$ & $9(18)$ & $21(42)$ & 6 \\
\hline \multicolumn{8}{|l|}{ U.K. } \\
\hline & Ozawa et al. & 2004 & 100 & $17(17)$ & $34(34)$ & $49(49)$ & 5 \\
\hline
\end{tabular}

U.K., United Kingdom.

OPC region, while British cases tended to show that the basal ganglia were more severely affected than the OPC region (Fig. 2). ${ }^{15)}$ They concluded that the OPC-predominant pathology was significantly more frequent in the Japanese series (40\%) than in the British series (17\%) (Table 2). ${ }^{6}$ )

\section{Discussion}

We reviewed articles detailing the natural history or epidemiology of MSA to determine the relative frequencies of MSA-P and MSA-C in various populations. At this point, it is evident that the majority of patients in studies from Asia exhibited MSA-C, whereas MSA-P predominated in studies from Europe and North America.

Only a few articles that addressed the relative prevalence of OPC- and SN-predominant cases in different populations were found during the systematic review of MSA pathology. The comparative study of MSA pathology in two large patient cohorts mentioned above ${ }^{5), 6)}$ demonstrated that the OPCpredominant pathology seems more frequent in Japanese MSA than in British MSA, and this finding is consistent with the tendency for MSA-C patients 


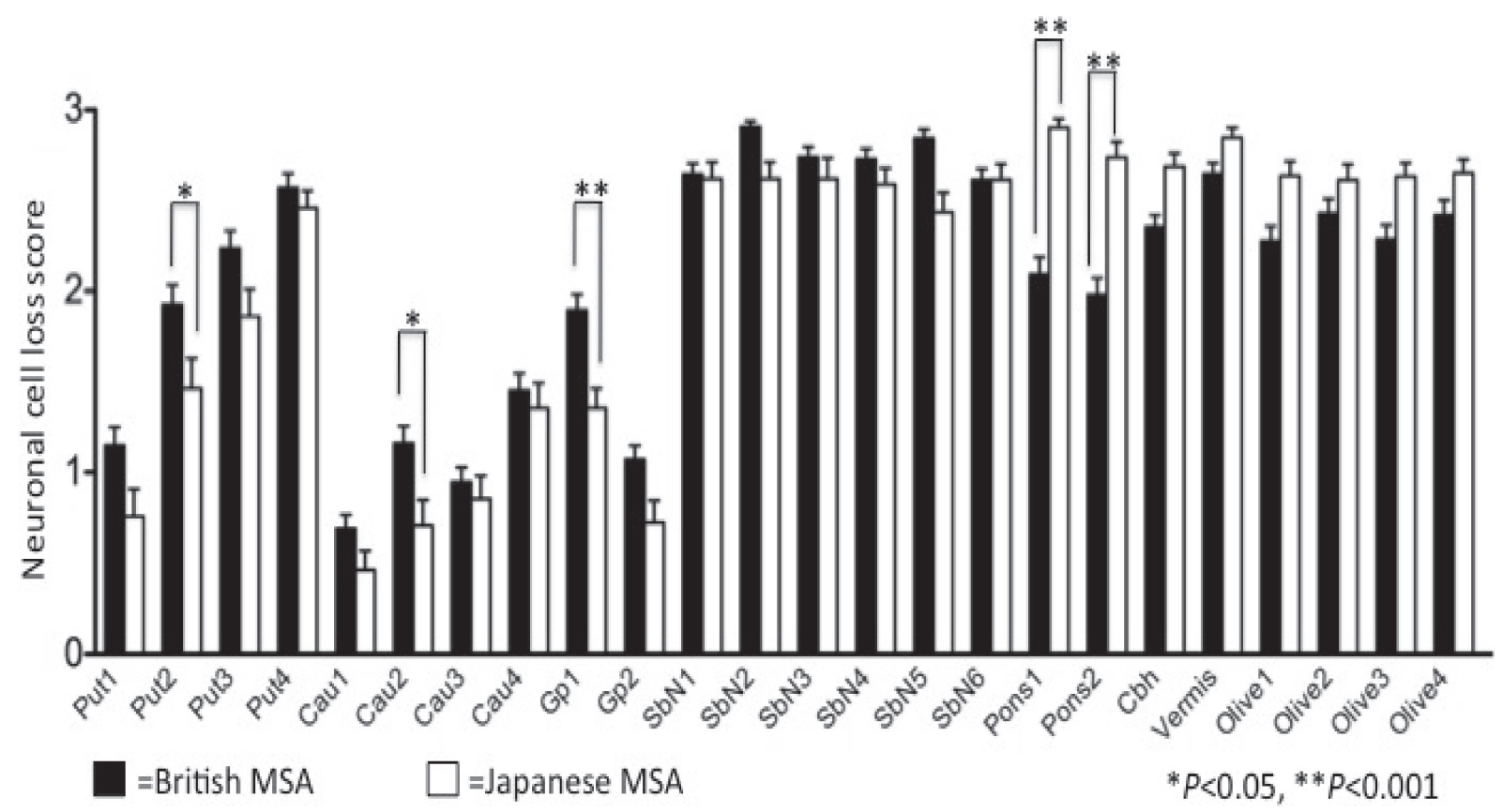

Fig. 2. Summary of the results of a comparative study of multiple system atrophy (MSA) cohorts carried out in the U.K. and Japan. British MSA patients have significantly higher scores for neuronal loss in Put2, Cau2, and GP1 than do Japanese patients $(P<0.05$, Bonferroni corrected). In Pons1 and 2, Japanese MSA patients have significantly higher scores for neuronal loss than British patients $(P<0.001$, Bonferroni corrected). Generally, British MSA cases tended to feature greater involvement of the basal ganglia, while Japanese cases tended to feature greater involvement of the olivopontocerebellar region. The substantia nigra is equally vulnerable in both British and Japanese patients with MSA. Put = putamen, Cau = caudate nucleus, GP = globus pallidus, $\mathrm{SbN}=$ substantia nigra, $\mathrm{Cbh}=$ cerebellar hemisphere. Data are represented as mean \pm standard error of the mean. This figure was reprinted from the reference no. 15, with permission from IOS Press.

to be more frequent in Japanese populations than in European populations (in Europe and North America). This points to a need for further investigations to elucidate biological factors contributing to regional differences in distributions of clinicopathological phenotypes of MSA.

In this review, whether the vulnerability of the autonomic nervous system in MSA differs across populations remains to be elucidated. Current diagnostic criteria for MSA indicate that autonomic dysfunction appearing as findings such as orthostatic hypotension or urinary disturbance is a sign essential for the clinical diagnosis of MSA. ${ }^{1)}$ Furthermore, autonomic dysfunction has been recognized as a predictor of poor prognosis in patients with MSA. ${ }^{16)}$ However, the severity of autonomic dysfunction has relatively less impact on the classification of clinical subtypes of MSA, so patients with prominent autonomic dysfunction are categorized into MSA-C or MSA-P groups in accordance with the relative dominance of cerebellar ataxia or parkinsonism. Criteria for evaluating the relative strength of autonomic dysfunction are thus needed to determine clinical subtypes of MSA that exhibit predominantly autonomic dysfunction. Endeavors to identify MSA patients with predominant autonomic dysfunction may provide basic sources of data to elucidate whether the vulnerability of the autonomic nervous system in MSA differs across populations.

MSA occurs sporadically, and familial cases of MSA are very rare. MSA is thus considered a sporadic disorder that involves multiple genetic and environmental factors that play roles at the molecular level. This concept raises the question of whether the genetic and environmental backgrounds for the development of MSA vary between populations. Because MSA is categorized as a synucleinopathy, as with Parkinson's disease, ${ }^{17)}$ genome-wide association studies (GWAS) have been performed to evaluate associations of the $\alpha$-synuclein gene with MSA. The most recent GWAS enrolled 918 patients of European ancestry with MSA, but demonstrated no association between the $\alpha$-synuclein gene and MSA. ${ }^{18)}$ This negative result from the largest GWAS was in keeping with a previous study by Ozawa et al., ${ }^{19)}$ which examined single nucleotide polymor- 
phisms in patients of European ancestry with MSA. In a Japanese population, mutations in the coenzyme Q2 (COQ2) gene were identified in multiplex families and sporadic cases of MSA using a combination of linkage analysis and whole-genome sequencing. ${ }^{20)}$ COQ2 encodes parahydroxybenzoate polyprenyl transferase, an enzyme involved in the biosynthesis of coenzyme $\mathrm{Q}_{10}$. Tracing a path through the discovery of COQ2 mutations, the MSA Research Collaboration performed linkage analyses on six multiplex families with Japanese MSA, and wholegenome sequencing on a member of a multiplex family in whom the diagnosis of MSA had been confirmed at autopsy. The results showed a homozygous mutation and compound heterozygous mutations in COQ2 were identified in two multiplex families. Extending the mutational analysis of COQ2 to sporadic case series of MSA, including 363 patients in a Japanese series, 223 patients in a European series, and 172 patients in a North American series, a common variant (V393A) was detected in Japanese MSA, but not in European or North American MSA. Furthermore, in the Supplementary Material of the paper, the ratio of MSA-C to MSA-P was significantly higher among carriers of $C O Q 2$ variants than among non-carriers. In the most recent GWAS mentioned above, no association was apparent between COQ2 and 918 patients of European ancestry with $\left.\mathrm{MSA},{ }^{18}\right)$ indicating that $C O Q 2$ variants contribute little to the development of MSA in European populations. In the Japanese population, the V393A allele was identified in 35 of 726 alleles $(4.8 \%)$ from MSA patients, ${ }^{20)}$ so the contribution of COQ2 variants to disease development remains within a minority of Japanese patients with MSA-C. These observations suggest that COQ2 variants in addition to many other genetic or environmental factors that are yet to be discovered may underlie the increased frequency of the features of MSA-C in Japanese patients. This notion points to the need for further investigations to determine genetic or environmental factors responsible for the increased frequency of MSA-C in Japanese patients.

Regarding environmental factors for MSA, since an occupational history of farming has been reported to increase the risk of MSA in European populations, ${ }^{21)}$ whether occupational exposure to pesticides was associated with European MSA has attracted considerable attention. However, subsequent studies found no associations between occupational exposure to pesticides and European patients with MSA. ${ }^{22), 23)}$ In Asian countries, occupational histories of farming or exposure to pesticides likewise have not been found to be associated with risk of MSA in a Korean cohort, in which MSA-C patients accounted for $73 \% .^{10)}$ Further epidemiological studies are needed to provide clues on possible environmental factors that contribute to the differences demonstrated in clinical and pathological subtypes of MSA in different populations.

\section{Conclusion}

To determine differences in clinical and pathological characteristics of MSA between ethnic groups or populations, we performed systematic reviews of studies that examined the relative frequencies of clinical or pathological subtypes of MSA in various populations. The result showed that the majority of MSA patients diagnosed in Japan and Korea have MSA-C, whereas the majority of MSA patients diagnosed in Europe and North America have MSA-P. A comparative study of MSA pathology with an identical methodological approach in British and Japanese cohorts demonstrated a difference in the distribution of phenotypes between the two populations. The frequent observation of the OPCpredominant pathology in Japanese cases of MSA is in keeping with previous findings that the majority of Japanese patients exhibit MSA-C. The OPCpredominant pathology and its clinical phenotype, MSA-C, thus represents the clinicopathological characteristics of Japanese patients with MSA. To date, genetic risk variants such as $C O Q 2$ variants are considered to play a role in the features of clinicopathological characteristics in Japanese patients with MSA. Environmental factors determining the predominating involvement of OPC versus SN regions in MSA remain to be determined. Further investigation is needed to determine the environmental and genetic factors that account for the differences demonstrated in clinicopathological phenotypes of MSA among different populations.

\section{Acknowledgements}

The Fig. 1 and the Fig. 2 were reprinted from "Ozawa, T., Revesz, T., Paviour, D., Lees, A., Quinn, N., Tada, M., Kakita, A., Onodera, O., Wakabayashi, K., Takahashi, H., Nishizawa, M. and Holton, J. (2012) Difference in MSA phenotype distribution between populations: genetics or environment? J. Parkinsons Dis. 2, 7-18." with permission from IOS Press. The publication is available at IOS Press through http://dx.doi.org/10.3233/JPD-201211056. 


\section{References}

1) Gilman, S., Wenning, G.K., Low, P.A., Brooks, D.J., Mathias, C.J., Trojanowski, J.Q., Wood, N.W., Colosimo, C., Durr, A., Fowler, C.J., Kaufmann, H., Klockgether, T., Lees, A., Poewe, W., Quinn, N., Revesz, T., Robertson, D., Sandroni, P., Seppi, K. and Vidailhet, M. (2008) Second consensus statement on the diagnosis of multiple system atrophy. Neurology 71, 670-676.

2) Graham, J.G. and Oppenheimer, D.R. (1969) Orthostatic hypotension and nicotine sensitivity in a case of multiple system atrophy. J. Neurol. Neurosurg. Psychiatry 32, 28-34.

3) Papp, M.I., Kahn, J.E. and Lantos, P.L. (1989) Glial cytoplasmic inclusions in the CNS of patients with multiple system atrophy (striatonigral degeneration, olivopontocerebellar atrophy and Shy-Drager syndrome). J. Neurol. Sci. 94, 79-100.

4) Wakabayashi, K., Yoshimoto, M., Tsuji, S. and Takahashi, H. (1998) Alpha-synuclein immunoreactivity in glial cytoplasmic inclusions in multiple system atrophy. Neurosci. Lett. 249, 180-182.

5) Ozawa, T., Paviour, D., Quinn, N.P., Josephs, K.A., Sangha, H., Kilford, L., Healy, D.G., Wood, N.W., Lees, A.J., Holton, J.L. and Revesz, T. (2004) The spectrum of pathological involvement of the striatonigral and olivopontocerebellar systems in multiple system atrophy: clinicopathological correlations. Brain 127, 2657-2671.

6) Ozawa, T., Tada, M., Kakita, A., Onodera, O., Ishihara, T., Morita, T., Shimohata, T., Wakabayashi, K., Takahashi, H. and Nishizawa, M. (2010) The phenotype spectrum of Japanese multiple system atrophy. J. Neurol. Neurosurg. Psychiatry 81, 1253-1255.

7) Gilman, S., Low, P.A., Quinn, N., Albanese, A., Ben-Shlomo, Y., Fowler, C.J., Kaufmann, H., Klockgether, T., Lang, A.E., Lantos, P.L., Litvan, I., Mathias, C.J., Oliver, E., Robertson, D., Schatz, I. and Wenning, G.K. (1999) Consensus statement on the diagnosis of multiple system atrophy. J. Neurol. Sci. 163, 94-98.

8) Yabe, I., Soma, H., Takei, A., Fujiki, N., Yanagihara, T. and Sasaki, H. (2006) MSA-C is the predominant clinical phenotype of MSA in Japan: analysis of 142 patients with probable MSA. J. Neurol. Sci. 249, 115-121.

9) Watanabe, H., Saito, Y., Terao, S., Ando, T., Kachi, T., Mukai, E., Aiba, I., Abe, Y., Tamakoshi, A., Doyu, M., Hirayama, M. and Sobue, G. (2002) Progression and prognosis in multiple system atrophy: an analysis of 230 Japanese patients. Brain 125, 1070-1083.

10) Seo, J.H., Yong, S.W., Song, S.K., Lee, J.E., Sohn, Y.H. and Lee, P.H. (2010) A case-control study of multiple system atrophy in Korean patients. Mov. Disord. 25, 1953-1959.

11) Kollensperger, M., Geser, F., Ndayisaba, J.P., Boesch, S., Seppi, K., Ostergaard, K., Dupont, E., Cardozo, A., Tolosa, E., Abele, M.
Klockgether, T., Yekhlef, F., Tison, F., Daniels, C., Deuschl, G., Coelho, M., Sampaio, C., Bozi, M., Quinn, N., Schrag, A., Mathias, C.J., Fowler, C., Nilsson, C.F., Widner, H., Schimke, N., Oertel, W., Del Sorbo, F., Albanese, A., Pellecchia, M.T., Barone, P., Djaldetti, R., Colosimo, C., Meco, G., Gonzalez-Mandly, A., Berciano, J., Gurevich, T., Giladi, N., Galitzky, M., Rascol, O., Kamm, C., Gasser, T., Siebert, U., Poewe, W. and Wenning, G.K. (2010) Presentation, diagnosis, and management of multiple system atrophy in Europe: Final analysis of the European multiple system atrophy registry. Mov. Disord. 25, 2604-2612.

12) Wenning, G.K., Geser, F., Krismer, F., Seppi, K., Duerr, S., Boesch, S., Kollensperger, M., Goebel, G., Pfeiffer, K.P., Barone, P., Pellecchia, M.T., Quinn, N.P., Koukouni, V., Fowler, C.J., Schrag, A., Mathias, C.J., Giladi, N., Gurevich, T., Dupont, E., Ostergaard, K., Nilsson, C.F., Widner, H., Oertel, W., Eggert, K.M., Albanese, A., del Sorbo, F., Tolosa, E., Cardozo, A., Deuschl, G., Hellriegel, H., Klockgether, T., Dodel, R., Sampaio, C., Coelho, M., Djaldetti, R., Melamed, E., Gasser, T., Kamm, C., Meco, G., Colosimo, C., Rascol, O., Meissner, W.G., Tison, F. and Poewe, W. (2013) The natural history of multiple system atrophy: a prospective European cohort study. Lancet Neurol. 12, 264-274.

13) Low, P.A., Reich, S.G., Jankovic, J., Shults, C.W., Stern, M.B., Novak, P., Tanner, C.M., Gilman, S., Marshall, F.J., Wooten, F., Racette, B., Chelimsky, T., Singer, W., Sletten, D.M., Sandroni, P. and Mandrekar, J. (2015) Natural history of multiple system atrophy in the USA: a prospective cohort study. Lancet Neurol. 14, $710-719$

14) Gatto, E., Rodriguez-Violante, M., Cosentino, C., Chana-Cuevas, P., Miranda, M., Gallin, E., Etcheverry, J.L., Nunez, Y., Parisi, V., Persi, G., Vecchi, C., Sanguinetti, A., Alleva, A., Aparcana, J., Torres, L. and Litvan, I. (2014) PanAmerican Consortium of Multiple System Atrophy (PANMSA). A Pan-American multicentre cohort study of multiple system atrophy. J. Parkinsons Dis. 4, 693-698.

15) Ozawa, T., Revesz, T., Paviour, D., Lees, A., Quinn, N., Tada, M., Kakita, A., Onodera, O., Wakabayashi, K., Takahashi, H., Nishizawa, M. and Holton, J. (2012) Difference in MSA phenotype distribution between populations: genetics or environment? J. Parkinsons Dis. 2, 7-18.

16) Tada, M., Onodera, O., Ozawa, T., Piao, Y.S., Kakita, A., Takahashi, H. and Nishizawa, M. (2007) Early development of autonomic dysfunction may predict poor prognosis in patients with multiple system atrophy. Arch. Neurol. 64, 256260.

17) Galvin, J.E., Lee, V.M. and Trojanowski, J.Q. (2001) Synucleinopathies: clinical and pathological implications. Arch. Neurol. 58, 186-190.

18) Sailer, A., Scholz, S.W., Nalls, M.A., Schulte, C., Federoff, M., Price, T.R., Lees, A., Ross, O.A., 
Dickson, D.W., Mok, K., Mencacci, N.E., Schottlaender, L., Chelban, V., Ling, H., O'Sullivan, S.S., Wood, N.W., Traynor, B.J., Ferrucci, L., Federoff, H.J., Mhyre, T.R., Morris, H.R., Deuschl, G., Quinn, N., Widner, H., Albanese, A., Infante, J., Bhatia, K.P., Poewe, W., Oertel, W., Hoglinger, G.U., Wullner, U., Goldwurm, S., Pellecchia, M.T., Ferreira, J., Tolosa, E., Bloem, B.R., Rascol, O., Meissner, W.G., Hardy, J.A., Revesz, T., Holton, J.L., Gasser, T., Wenning, G.K., Singleton, A.B., Houlden, H., European Multiple System Atrophy Study Group and the UK Multiple System Atrophy Study Group (2016) A genome-wide association study in multiple system atrophy. Neurology 87, 1591-1598.

19) Ozawa, T., Healy, D.G., Abou-Sleiman, P.M. Ahmadi, K.R., Quinn, N., Lees, A.J., Shaw, K., Wullner, U., Berciano, J., Moller, J.C., Kamm, C., Burk, K., Josephs, K.A., Barone, P., Tolosa, E., Goldstein, D.B., Wenning, G., Geser, F., Holton, J.L., Gasser, T., Revesz, T. and Wood, N.W. (2006) The alpha-synuclein gene in multiple system atrophy. J. Neurol. Neurosurg. Psychiatry
77, 464-467.

20) The Multiple-System Atrophy Research Collaboration (2013) Mutations in COQ2 in familial and sporadic multiple-system atrophy. N. Engl. J. Med. 369, 233-244.

21) Vanacore, N., Bonifati, V., Fabbrini, G., Colosimo, C., De Michele, G., Marconi, R., Stocchi, F., Nicholl, D., Bonuccelli, U., De Mari, M., Vieregge, P. and Meco, G. (2005) Case-control study of multiple system atrophy. Mov. Disord. 20, $158-163$.

22) Chrysostome, V., Tison, F., Yekhlef, F., Sourgen, C., Baldi, I. and Dartigues, J.F. (2004) Epidemiology of multiple system atrophy: a prevalence and pilot risk factor study in Aquitaine, France. Neuroepidemiology 23, 201-208.

23) Vidal, J.S., Vidailhet, M., Elbaz, A., Derkinderen, P., Tzourio, C. and Alperovitch, A. (2008) Risk factors of multiple system atrophy: a case-control study in French patients. Mov. Disord. 23, 797803.

(Received Jan. 6, 2017; accepted Jan. 30, 2017)

\section{Profile}

Tetsutaro Ozawa was born in Aizu-Wakamatsu City, Fukushima Prefecture in 1965. He graduated from Niigata University School of Medicine in 1991. He majored in Neurology at Brain Research Institute, Niigata University. He received Ph.D. degree in molecular genetics of neurodegenerative disease at Niigata University in 2001. From 2002 to 2003, he worked as a Research Fellow at the UCL Institute of Neurology and the Queen Square Brain Bank in London, U.K., to investigate the pathogenesis of neurodegenerative diseases. He also worked as a Specialist Clinical Fellow at the National Hospital for Neurology \& Neurosurgery in London. During his stay in U.K., he made considerable achievements in the research for elucidating the spectrum of pathological involvement of striatonigral and olivopontocerebellar system in multiple

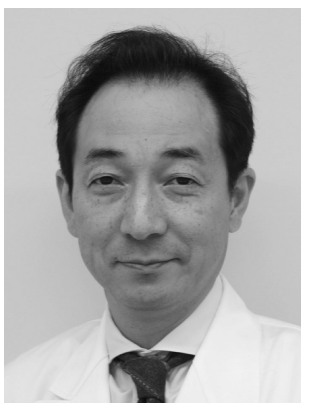
system atrophy. He worked as a Research Associate (from 2004 to 2008) and a Lecturer (from 2009 to 2014) in the Department of Neurology, Brain Research Institute, Niigata University. He was appointed Editorial Board Member of Acta Neuropathologica (Springer) from 2008 to 2010. He was also appointed Editorial Board Member of Movement Disorders (the official journal of the International Parkinson and Movement Disorder Society) from 2010 to 2012. For his accomplishment, he received the Yujin Young Investigators Award (from Niigata University), the Kurt Jellinger Prize (from the German Society for Neuropathology and Neuroanatomy), and Outstanding Dedication and Service as Editorial Board Member of Movement Disorders (from the International Parkinson and Movement Disorder Society). He is currently a Professor of Neurology at the Uonuma Institute of Community Medicine, Niigata University Medical and Dental Hospital. 